
\title{
$\begin{array}{ll}\text { Research Square } & \text { They should not be considered conclusive, used to inform clinical practice, } \\ \text { or referenced by the media as validated information. }\end{array}$
}

\section{Generation and Evaluation of Chicken IgY-scFv for the Purposes of CPV Diagnosis and Therapy}

Shikun Ge

"Universidade do Minho"

Long Xu

"Universidade do Minho"

Ben Li

Shannxi University of Technology

Fagang Zhong

Xinjiang Academy of Environmental Protection Science

XiaoYing Zhang ( $\sim$ zhang@bio.uminho.pt)

"Universidade do Minho"

\section{Research}

Keywords: Chicken IgY single chain variable fragments (IgY-scFv), Canine parvovirus (CPV), T7 phage display system, Virus-like particles (VLP), novel antibody generation platform

Posted Date: May 19th, 2020

DOI: https://doi.org/10.21203/rs.3.rs-28773/v1

License: (c) (1) This work is licensed under a Creative Commons Attribution 4.0 International License. Read Full License 


\section{Abstract}

Background: Canine parvovirus (CPV) can cause acute and highly contagious enteritis in dog, antibodies have been used for diagnosis and therapy of CPV disease, generation of functional antibody fragments by using novel antibody engineering platforms is promising in veterinary practice.

Results: The IgY single chain fragment variables (scFv) were generated by T7 phage display system and expressed in E. coli system after immunizing hens with virus-like particles (VLP) of CPV-VP2. The titer of the primary scFv library reached to $1.5 \times 106 \mathrm{pfu} / \mathrm{mL}$, and $95 \%$ of the phages contained the target fragments. The CPV-VLP and CPV-VP2 protein showed similar reaction values to the purified scFv in the ELISA test, and the results of ELISA analysis using IgY-scFv toward CPV clinical samples were consistent with commercial immunochromatographic assay (ICA) and PCR detection, the scFv did not show cross reactivity with canine distemper virus (CDV) and canine coronavirus (CCV). IgY-scFv was successfully expressed in CRFK cells, and in the virus suppression assay, $55 \%$ of CPV infections were eliminated within 24 hours. Docking results demonstrated that the number of amino acids of the binding sides between scFv and VP2 were AA37 and AA40, respectively.

Conclusions: This study revealed the feasibility of a novel functional antibody fragment development strategy by generating diversified avian IgY-scFv libraries towards the pathogenic target of interest for both detection and therapeutic purposes in veterinary medicine.

\section{Background}

Canine parvovirus (CPV) was first identified in 1978 (1); it has a single-stranded DNA genome of negative polarity, about 5200 bp in length. The CPV capsid is a $25 \mathrm{~nm}$ diameter icosahedron containing three structural viral proteins (VP1, VP2 and VP3) and two non-structural proteins (NS1 and NS2), among them, VP2 accounts for $90 \%$ of the viral capsid and represents the major determinant of host range and virus-host interactions (2). Although the conventional attenuated and inactivated CPV vaccines have been successful in reducing the disease outbreaks, the genus of parvovirus still causes severe epizootics worldwide and leads to severe economic losses in dogs (3).

Antibody based approach is promising in CPV disease diagnosis, therapy and prevention, and the relevant hyper immune serum (IgG) and full-length monoclonal antibody (mAbs) have been routinely applied in the veterinary practices (4). At present, most commercially available mAbs are produced in mammalian system using hybridoma technology. Functional antibody fragments (i.e.: scFv, Fab) generated by phage-display technology have been not yet routinely evaluated and applied in the veterinary medicine despite it has been well confirmed that such engineered antibodies offer a series of advantages over polyclonal and full-length monoclonal antibodies. Antibody phage display technology is to display polyclonal antibodies on the surface of the phage shell protein and to screen the specific monoclonal antibodies by bio-panning procedure (5). Phages are more stable and can be stored for years at $4{ }^{\circ} \mathrm{C}$; it can be reproduced rapidly, successively and inexpensively with a confirmed sequence in the prokaryote or eukaryote systems without hybridoma and immunization procedure. Furthermore, higher affinity mutants of scFv can be generated through site directed mutagenesis which is much easier and simpler to be performed (6).

Chicken (Gallus domesticus) IgY antibody generated by lgY technology is another conventional approach in generating large amount of high specific antibody and have been used for broad biomedical purposes owning to a series of advantages such as higher productivity, better animal welfare, higher immunogenicity to mammal conserved proteins and lower cross-reactivity, as compared to the generation and application of mammalian serum IgG (7). Our previous work confirmed that the generated polyclonal IgY targeted to CPV-VP2 could applied into the immunotherapy and immunoprophylaxis for the CPV infection (8). In recent years, as an interesting tendency in IgY technology, generation of IgY-scFv in order to better combine the biological superiorities of both $\lg \mathrm{Y}$ and recombinant antibody fragment, is gaining increasing attention and application $(9,10)$. Our previous studies demonstrated that IgY-scFv can be generated and applied in different immunoassays for the detection of small molecules gentamicin (11) and large molecules pIFN-y (12) in the veterinary practice.

As an attempt to understand the feasibility of chicken IgY-scFv in diagnosis and therapy of veterinary diseases, this study aimed to construct and characterize chicken sourced ScFv against CPV-VP2 virus like particles (VLP) using phage display technology, and to evaluate the specificity, sensitivity and virus inhibition ability of the obtained scFv.

\section{Methods}

\section{Immunization of chicken}

Twelve-week old white Leghorn hens were immunized intramuscularly with CPV-VLP (provided by Dr. Shiqi Sun (13)) mixed with Freund's adjuvant (SigmaAldrich, St. Louis, MO, USA) at four different sites of breast muscles. CPV-VLP protein ( $125 \mu \mathrm{L}, 2 \mathrm{mg} / \mathrm{mL})$ in equal volume of phosphate buffered solution (PBS, $0.01 \mathrm{M}, \mathrm{pH}$ 7.4) was emulsified with Freund's complete adjuvant (FCA; Sigma-Aldrich, St. Louis, MO, USA) in the first immunization, and four booster immunizations were followed up using Freund's incomplete adjuvant (FIA; Sigma-Aldrich, St. Louis, MO, USA) at 2-week intervals. All experimental animal protocols were reviewed and approved by the institutional Ethics Committee for the use of laboratory animals.

\section{Construction Of Anti-cpv-vlp Scfv}

The hen's spleen was collected to extract the total RNA using Total RNA Kit (Tiangen Biotech, Beijing, China), and the first-strand cDNA was synthesized using HiScript Q Select RT SuperMix for PCR (+ gDNA wiper, Vazyme Biotech, Nanjing, China). The heavy variable fragment $\left(V_{H}\right)$ and light chain variable fragment 
$\left(V_{L}\right)$ genes were amplified by PCR with primers (Table 1). The $V_{H}$ and $V_{L}$ were assembled using primers HF-EcoR I \& LR-Hind III by Overlap PCR. The products of overlap PCR (scFv) were purified through Gel Extraction Kit (Omega, Norcross, GA, USA).

Table 1

Primers used for PCR

\begin{tabular}{|c|c|c|}
\hline Name & Primer sequences $\left(5^{\prime}-3^{\prime}\right)$ & Application \\
\hline HF-EcoR I & CGGAATTCGGCCGTGACGTTGGACG & $\mathrm{V}_{\mathrm{H}}$ \\
\hline \multirow[t]{2}{*}{ HR-Linker } & CAGAGCCACCTCCGCCTGAACCGCCTCCA & \multirow[t]{2}{*}{$\mathrm{V}_{\mathrm{H}}$} \\
\hline & CCGGAGGAGACGATGAC & \\
\hline \multirow[t]{2}{*}{ LF-Linker } & TTCAGGCGGAGGTGGCTCTGGCGGTGGCG & \multirow[t]{2}{*}{$V_{L}$} \\
\hline & GATCGGCGCTGACTCAGCCGTCCT & \\
\hline LR-Hind III & AATAAGCTTACCTAGGACGGTCAGGG & $\mathrm{V}_{\mathrm{L}}$ \\
\hline
\end{tabular}

\section{Construction Of Library}

The scFv gene products were digested with EcoR I and Hind III restriction enzyme and ligated to T7 select 10-3b (0.04 pmol; Novagen, Darmstadt, Germany) using T4 DNA Ligase (TaKaRa Biotechnology, Dalian, China) in a working volume of $5 \mu \mathrm{L}$ at $16^{\circ} \mathrm{C}$ overnight. The ligation products were directly added to T7 packaging extract $(25 \mu \mathrm{L})$ and the mixtures were incubated at $22^{\circ} \mathrm{C}$ for $2 \mathrm{~h}$ in vitro packaging to create the primary library by adding $\mathrm{LB}$ medium ( $\left.270 \mu \mathrm{L}\right)$ to stop the reaction. The primary scFv library was amplified by liquid lysate amplification according to the T7 select system manual. The titers of the primary and amplified library were evaluated by plaque assay.

\section{Bio Panning}

The amplified library was subjected to four rounds of bio panning on microplates for the enrichment of the specific scFv phages according to the T7 select system manual. The microplates were coated with CPV-VLP overnight $\left(15 \mu \mathrm{g}, 10 \mu \mathrm{g}, 8 \mu \mathrm{g}\right.$ and $5 \mu \mathrm{g}$ per well in each round) at $4^{\circ} \mathrm{C}$, and washed with TweenPBS (PBST, tween $0.5 \% ; 150 \mu \mathrm{L} /$ well) and PBS, respectively. After blocked with skimmed milk (5\%) in PBST, the amplified phages from the starting library of each round of panning were added to the microplates and incubated at $37^{\circ} \mathrm{C}$ for $1 \mathrm{~h}$, and the bound phage was eluted with SDS (1\%) in each round. In the first three rounds, eluted phages were amplified by infecting BL5403 bacterial culture (Novagen, Darmstadt, Germany). The enrichment of specificity was determined from the input/output ration of the phage. A total of random clones of 100 on the plates were selected from the fourth round to identify the positive rate using a pair of universal primers (Table 1). The amplified library was added with $1 \%$ chloroform and stored at $4^{\circ} \mathrm{C}$.

\section{Expression And Purification Of Scfv}

After sequencing, the phage-scFv with the highest binding force was selected to ligate with pET-30a (+) vector using T4 DNA Ligase (TaKaRa Biotech, Dalian, China). The recombinant plasmids were transformed into BL21 (DE3) competent cells, and the scFv expression was induced with isopropyl- $\beta$-D-

thiogalactopyranoside (IPTG). The bacterial cells were collected by centrifugation and sonication, and the supernatants and pellets were used for SDS-PAGE analysis, respectively. Protein was purified by HisTrap HP histidine-tagged protein cultured columns (GE, Pittsburgh, PA, USA).

\section{Identification Of Scfv}

The CPV-VP2 proteins were separated by SDS-PAGE (5\% stacking and 12\% resolving) and blotted on nitrocellulose membranes (Schleicher \& Schuell, Atlanta, GA, USA). Membranes were blocked with $5 \%(w / v)$ nonfat dry milk (NDM) in Tris-buffered saline-Tween (TBS-T) at room temperature (RT) for 30 min. Membranes were incubated with $12.5 \mathrm{~mL}$ TBS-T containing $1 \%$ NDM and $5 \mu \mathrm{g}$ prepared scFv proteins at RT under agitation for $1 \mathrm{~h}$. Membranes were washed three times for $10 \mathrm{~min}$ in TBS-T, and incubated with $12.5 \mathrm{~mL}$ TBS-T containing 1\% NDM and mouse anti-His monoclonal antibody conjugated with HRP (1:5000; CWBio, Beijing, China) at RT under agitation for 30 min. Membranes were washed three times for 15 min in TBS-T, and developed using DAB chromogenic solution detection reagents (Boster, Wuhan, China).

\section{Analysis On Scfv Sensitivity By Elisa}

The wells of a 96-well Maxisorp microtiter plate (Nunc, Roskilde, Denmark) were coated with different amounts of CPV-VP2 (0, 0.2, 0.5, 2, 6, 8, 10 ng/ $\mu \mathrm{L}$ ) dissolved in carbonate buffer $\left(15 \mathrm{mM} \mathrm{Na}_{2} \mathrm{CO}_{3}, 35 \mathrm{mM} \mathrm{NaHCO}_{3}, 0.001 \%\right.$ phenol-red, pH 9.6) and incubated over night at $4{ }^{\circ} \mathrm{C}$. The wells were rinsed with PBST and incubated with scFv protein (different amount) diluted in PBST for $1 \mathrm{~h}$. The wells were rinsed with PBST ( $3 \times 5 \mathrm{~min})$ and incubated $1 \mathrm{~h}$ with mouse anti-His monoclonal antibody conjugated with HRP (1:5000; CWBio, Beijing, China) followed by three washes with PBST buffer ( 5 min each). The color will be developed using 3, 3', 5, 5' tetramethylbenzidine (TMB; Promega Biotech, Beijing, China) for 10 min and the absorbance was read at $450 \mathrm{~nm}$. 


\section{Detection Of Clinical Samples}

To determine the accuracy of scFv, a total of 28 clinical dog stool samples were collected using sterile swabs in an animal hospital (Xinger, Xi'an, China). Among them, 24 samples were confirmed CPV positive and 4 were negative by the hospital using commercial colloidal gold test strip (ICA). The samples were homogenized $(10 \%, w / v)$ in PBS (1 mL, pH 7.2) and centrifuged, the supernatants were used for indirect ELISA and PCR. In order to verify whether scFv has specificity in clinical practice detection, Canine distemper virus (CDV) and coronavirus (CCV) were used to detect the cross-reaction by ELISA.

\section{Indirect Immunofluorescence Assay (ifa)}

The CRFK cells (LMAI Bio, Shanghai, China) were seeded into 6-well plates and pcMV-3-scFv were transfected when the cell density reached $70 \%$. At $48 \mathrm{~h}$ posttransfection, the cells were fixed with paraformaldehyde (4\%), permeabilized with Triton X-100 (0.5\%) and incubated with BSA (Beyotime, Shanghai, China) to block nonspecific binding sites. They were then incubated with diluted His-tag antibody (100 $\mu \mathrm{L}$; Affinity Biosciences, OH, USA) for $2 \mathrm{~h}$. After PBS washing, Goat anti-Mouse lgG $(\mathrm{H}+\mathrm{L}$ ) Fluor 594-conjugated (Affinity Biosciences, $\mathrm{OH}, \mathrm{USA}$ ) was added. The stained cells were visualized by using a Nikon Eclipse 80i fluorescence microscope (Nikon, Sendai, Japan). Total protein of the cells transfected $48 \mathrm{~h}$ were extracted for western blot; His-tag antibody and Goat antiMouse IgG HRP (Biosharp, Hefei, China) were used as primary and secondary antibodies, respectively.

\section{Measurement Of Virus Growth Curve}

Normal CRFK cells $\left(10^{5} / \mathrm{mL}\right)$ were seeded into 96 -well plates, and the virus infection was performed when the cell density reached $70 \%$. The original virus solution was serially diluted in 10 -fold, added to a 96-well plate, 10 gradients, and 8 wells were repeated for each gradient. The $96-$ well plate was placed in $37^{\circ} \mathrm{C}$ with $5 \% \mathrm{CO}_{2}$ incubator, and the tissue culture infective dose $\left(\mathrm{TCID}_{50}\right)$ was determined after the number of pathological wells was fixed.

Transfected cells and normal cells were seeded into 96-well plates, and the cells were infected with CPV (CPV-HY strain offered by Century Yuanheng Animal Epidemic Prevention Technology Co., Ltd., Beijing, China) in $0.1 \mathrm{MOI}$. After $1 \mathrm{~h}$, the supernatant virus solution was removed and replaced with a cell maintenance solution. The cell supernatants were collected at $2 \mathrm{~h}, 4 \mathrm{~h}, 8 \mathrm{~h}, 12 \mathrm{~h}, 24 \mathrm{~h}, 36 \mathrm{~h}, 48 \mathrm{~h}, 60 \mathrm{~h}, 72 \mathrm{~h}$ and $84 \mathrm{~h}$, respectively, and the TCID 50 values of each time point were measured to plot the growth curve.

\section{Scfv-vp2 Docking}

Docking was performed using Discovery Studio 4.5 software (BIOVIA, San Diego, CA, USA) and the Antibody Modeling Cascade program in Discovery Studio software was used to build the molecular model of the scFv. Then, to obtain the docking conformation, the scFv molecular model was docked with VP2 using the ZDock molecular docking program. Finally, the Residues Contact Frequency (RCF) algorithm was used to analyze the predicted results, and the binding surfaces of amino acids in ScFv and VP2 were obtained.

\section{Results}

\section{Construction of scFv library}

The lengths of VH-linker, VL-linker and scFv were approximately 370 bp, 420 bp and 800 bp, respectively (Fig. 1A and B). The titer of the primary anti-CPV scFv library (Fig. 1C) and the amplified library were $1.5 \times 10^{6} \mathrm{pfu} / \mathrm{mL}$, and $8.2 \times 10^{11} \mathrm{pfu} / \mathrm{mL}$, respectively. There were $95 \%$ of the phages containing the target fragments in the primary scFv library.

Notes: A: PCR products of VL (370 bp, lane 1 and 2) and VH (420 bp, lane 3 and 4); B: PCR products of scFv, 800 bp (lane 1). M: marker; C: schematic diagram of scFv construction.

\section{Screening Of Scfv Phage Library}

After 4 rounds of "bind-elute-amplify" bio-panning procedure, the data of input and output phages in each round indicated that the phage library had been enriched 100 times (Fig. 2 and Table 2). A total of 15 scFv genes showed relatively high binding capacity to CPV-VLP in the reactivity detection of 100 phages, and the sequencing confirmed that all the $15 \mathrm{scFv}$ genes have complementary determining region 3 (CDR3) that was the main mutation region in both $\mathrm{VH}$ and VL. There were few clones having limited mutations in framework region (FR, Fig. 3). The phage-scFv (No. 96) showed the highest binding force was chosen for further experiments. 
Table 2

Library size and phage titer of each panning

\begin{tabular}{|lllll|}
\hline Round & Coating concentration (per well) & $\begin{array}{l}\text { Input } \\
(\mathrm{pfu} / \mathrm{mL})\end{array}$ & $\begin{array}{l}\text { Output } \\
(\mathrm{pfu} / \mathrm{mL})\end{array}$ & Amplification library (pfu/mL) \\
\hline 1 & $15 \mu \mathrm{g}$ & $1 \times 10^{12}$ & $7.2 \times 10^{6}$ & $4.95 \times 10^{12}$ \\
\hline 2 & $10 \mu \mathrm{g}$ & $1 \times 10^{11}$ & $4.64 \times 10^{7}$ & $1.31 \times 10^{13}$ \\
\hline 3 & $8 \mu \mathrm{g}$ & $1 \times 10^{11}$ & $8.8 \times 10^{8}$ & $2.8 \times 10^{12}$ \\
\hline 4 & $5 \mu \mathrm{g}$ & $1 \times 10^{11}$ & $1.52 \times 10^{8}$ & - \\
\hline
\end{tabular}

\section{Note}

The yellow markers represented the same sequences.

\section{Scfv Expression And Purification}

The solubility analysis showed that scFv mainly existed in the form of inclusion body (38 kDa; Fig. 4A, lane 2). The denaturation and purification of scFv inclusion body protein showed a single protein band with high purity (Fig. 4B). The VP2 protein could bind to scFv with a single binding strip (85 kDa; Fig. 4C).

Notes: A: lane 1, E. coli supernatant; lane 2, E. coli precipitation; B: lane 1, the renaturation of scFv protein; C: lane 1, antigen was CPV-VP2 protein, incubated with primary antibody scFv.

\section{Scfv Sensitivity Analysis}

The immunoreactivity of scFv against soluble VP2 was examined by ELISA. scFv bound in a dose-dependent manner to the soluble VP2 (Fig. 5). The minimum antibody concentration for the detected antigen was $2 \mathrm{ng} / \mu \mathrm{L}$.

\section{Notes}

scFv in different concentrations were added to 96 -well plates coated with soluble VP2 in different concentrations $(0,0.2,0.5,2,6,8,10 \mathrm{ng} / \mu \mathrm{L})$. Binding was detected with mouse anti-His monoclonal antibody conjugated with HRP. The binding activity was measured as absorbance at $450 \mathrm{~nm}$ produced by peroxidase. Data of each point represented as Mean \pm SD of triplicate.

Specificity and cross-reactivity of IgY-scFv in CPV clinical sample tests

The coincidence of ELISA (Fig. 6A) and PCR (Fig. 6C) with ICA (data not show) was $100 \%$ and $85.7 \%$, respectively. The scFv showed no cross reactivity with CDV and CCV (Fig. 6B).

\section{Notes}

Clinical samples of CPV were analyzed by ELISA (A), PCR (C) and ICA (data not shown); the cross reactivities of anti-CPV-IgY-scFv with CDV and CCV were analyzed by ELISA (B). CPV was CPV-HY strain; VP2 was expressed in the prokaryotic system.

\section{Neutralization Effect Of Scfv To Cpv In Crfk Cells}

The sequencing results showed that the scFv and pcMV-3 vectors were successfully connected (Fig. 7A), IFA confirmed that scFv was significantly expressed in CRFK cells (Fig. 7B). Immunoblotting further confirmed that the scFv protein was correctly folded and modified in the cell (Fig. 7C). The CRFK cells expressing scFv showed a small amount of cytopathic effect (CPE) after CPV infection; the cells without scFv expression were broke away significantly from the bottom wall, became round, and some cells even broke up (Fig. 7D). Virus TCID $_{50}$ at different time points was determined; the growth rate of virus in cells expressing scFv was significantly lower than that in cells not expressing scFv (Fig. 7E). The inhibition rates of scFv on virus growth at $24 \mathrm{~h}, 48 \mathrm{~h}$, and $72 \mathrm{~h}$ were $55 \%, 38 \%$ and $30 \%$, respectively (Fig. 7 F).

Notes: A, scFv gene was ligated pcMV-3 vector mode. B, control: CRFK cells of pcMV-3 without scFv, scFv: CRFK cells of pcMV-3 with scFv, DAPI: staining nuclei. C, western blot detection scFv transfected CRFK cells, control represent empty pcMV-3 transfected CRFK cells. D, after transfecting the cells with scFv for $24 \mathrm{~h}$, the cells were infected with CPV $(\mathrm{MOI}=0.1)$. E, growth curve of CPV in CRFK cells with scFv and without scFv expression. $\mathrm{F}$, The proportion of TCID50 of virus in different cell treatment groups (scFv group and without scFv group) at different time points.

\section{The Binding Sides Of The Scfv-vp2}


The main structural domains of scFv were VLCDR1, VLCDR2, VLDR3, VHCDR1, VHDCR2, and VHCDR3, with 13 antigen-binding sites on scFv (Fig. 8A). The 3 dimensional scFv model was subsequently constructed (Fig. 8B). A total of 5 highly similar VP2 homologous sequences were simulated by software (Fig. 8C), the VP2 molecular stereo model was established by combining the characteristics of each sequence (Fig. 8D). After analysis on ScFv and VP2 binding mode (Fig. 8E), the interacting amino acids at the binding sides of ScFv (AA37) and VP2 (AA40) were confirmed (Fig. 8F and 8G).

Notes: All docking analysis were performed by Discovery Studio software. ScFv modeling: Antibody Modeling Cascade program; VP2 modeling: Build Homology Models program; Docking: ZDock program.

\section{Discussion}

The mAbs have been widely applied in the biomedical areas owning to their high specificity and homogeneity. However, mAbs produced by mammals may have the side effects of immunogenicity, thrombocytopenia and hypersensitivity reactions, etc, which has greatly limited the application of mAbs in target detection and treatment $(14,15)$. With the development of antibody library technology and humanized antibody modification technology, increasing humanized recombinant antibodies have been gradually developed and entered the clinic trials (16). Diversified antibody generation strategies could be a future tendency in antibody engineering in order to better combine the characteristics and advantages of antibodies from different sources. As a notable example, Brolucizumab (Beovu) is the first FDA approved rabbit-derived scFv used as vascular endothelial growth factor (VEGF) inhibitor for the treatment of exudative (wet) age-related macular degeneration (AMD), diabetic macular oedema and macular oedema secondary to retinal vein occlusion, which could better overcome the possible side effects (discomfort and increased tears in the affected eyes, itchy or watery eyes, dry eyes, swelling of the eyelids, etc.) of murine-derived IgG-Fab fragment (Lucentis) (17). In avian IgY, similar attempts have also been made. For instance, the snake venom contains neurotoxic proteins, the urgent administration of hyperimmune serum from horse used to be the most efficient treatment, which can recognize many different antigenic determinants. However, generation of equine anti- venom is costly and associated with several potential side effects. Chicken IgY-scFv has been generated against glutaraldehyde-attenuated Daboia russelii formosensis (DRF) venom proteins for passive immunization, which can identify and neutralize the toxic activity of the venom components, with only small quantity of antigens required to induce a significant antibody response in hens, and can be also used as a rapid diagnostic tool for wound secretions to determine snake types (18).

As summarized by previous authors, owning to the unique structure, phylogenetic distance and the mechanism of molecular diversification, IgY antibodies provide a series of important advantages over mammal lgG, including the stronger immune responses of chicken system to the proteins conserved among mammals, decreased/avoid cross-reactivities (ie.: rheumatoid factor, human anti-mouse lgG antibody, complement system, Fc receptors) in the mammal systems $(19,20)$, and more convenient to design a primer against IgY-scFv (Table 1), as IgY only has one isotype and it is lack of hinge region (9). Furthermore, it is noteworthy to address, recent studies confirm that from the glycobiology point of view, recombinant IgY antibody could be a potentially promising immune-therapeutic candidate after proper antibody engineering and expression as $\lg \mathrm{Y}$ is more heavily glycosylated (21), and has higher sialic acid content (22) as compared to mammal IgG. Recombinant functional antibody fragments remove or reduce irrelevant structures, while retain the specificity and main biological activities of natural antibodies, which offers a wider application prospect than natural antibodies (23). Recombinant IgY-scFv could combine the advantages of both IgY molecular and functional antibody fragment (9).

Designing on chimeric antibody could be the next step for IgY-scFv study in order to provide better compatibility of the antibody in the host system, and to recoup the possibly decreased specificity and affinity of antibody fragments as compared to full length antibody. Recent study confirmed that mammalian IgG and avian IgY shared compatible V-C region interfaces, which may be conducive for the design and utilization of mammalian-avian chimeric Abs (24).

In our study, the high consistency of ELISA analysis to PCR and ICA on clinical samples confirmed the specificity of the obtained IgY-scFv (Fig. 6), which offers the potential using IgY-scFv for rapid detection of CPV. ScFv neutralized the virus (Fig. 7D), inhibited CPV replication with significantly reduced growth rates of the CPV observed in the CRFK cells (Fig. 7E, F), which provides the value of further therapeutic investigation of obtained IgY-scFv.

As alternative to viral components, CPV-VP2-VLP was used as immunogen in this study. With increasing applications in vaccine design and immunization, VLP has been recognized as safe and effective particle to stimulate adequate immune responses for both viral and non-viral diseases by inducing lymphocyte proliferation and specific antibody with high titer (25). The docking of scFv-VP2 shows that there were 13 antigen-binding sites on scFv, with high binding force to VP2. According to Residues Contact Frequency (RCF) algorithm analysis, 37 binding amino acids on scFv and 40 binding amino acids on VP2 were involved in the binding (Fig. 8), these results provide us confidence that a well-designed CPV-VLP can be used as a potent immunogen to induce qualified specific antibodies.

In Conclusion, we demonstrated that specific IgY-scFv can be generated with high specificity and significant inhibition to CPV growth. As a preliminary evaluation, our work revealed the potential of IgY-scFv as a novel approach in veterinary diagnosis and therapy.

\section{Abbreviations}

\section{CPV}

canine parvovirus; VLP:virus-like particles; scFv:single chain fragment variables; ICA:immunochromatographic assay; CDV:canine distemper virus; CCV:coronavirus; IgG:immune serum; mAbs:monoclonal antibody; IgY-scFv:Chicken IgY single chain variable fragments; IPTG:isopropyl-ß-Dthiogalactopyranoside

\section{Declarations}


Dr. XY Zhang acknowledges the adjunct position and supports offered by the University of Guelph, Canada.

\section{Funding}

This work was finically supported by the grand of China Natural Science Foundation $(31873006,31572556)$ and the Incubation Project on State Key Laboratory of Biological Resources and Ecological Environment of Qinba Areas (SLGPT2019KF04-04).

\section{Availability of data and materials}

All data supporting our findings are included in the manuscript.

\section{Authors' contributions}

$X Y Z, F G Z$ and SKG conceived this project. SKG, LX and BL performed the experiments. SKG, LX and XYZ wrote the manuscript. All authors read and approved t

\section{Ethics approval and consent to participate}

Not applicable.

\section{Consent for publication}

Not applicable.

\section{Competing interests}

The authors declare that they have no competing interests.

\section{References}

1. Thomson GW, Gagnon AN. Canine gastroenteritis associated with a parvovirus-like agent. The Canadian veterinary journal = La revue veterinaire canadienne. 1978;19(12):346.

2. Tsao J, Chapman M, Agbandje M, Keller W, Smith K, Wu H, et al. The Three-Dimensional Structure of Canine Parvovirus and Its Functional Implications. 251. New York: Science; 1991. pp. 1456-64.

3. Hu J-J, Zhang X, Han S-Z, Zhao J-Z, Tian Z-H. A Survey of Diagnosis and Treatment of Pet Canine Parvovirus Disease in China. Journal of Animal Veterinary Advances. 2011;10:2058-60.

4. Braganza A, Wallace K, Pell L, Parrish CR, Siegel DL, Mason NJ. Generation and validation of canine single chain variable fragment phage display libraries. Vet Immunol Immunopathol. 2011;139(1):27-40.

5. Kotlan B, Glassy MC. Antibody phage display: overview of a powerful technology that has quickly translated to the clinic. Methods in molecular biology (Clifton NJ). 2009;562:1-15.

6. Ma H, O'Kennedy R. Recombinant antibody fragment production. Methods (San Diego, Calif). 2017;116:23-33.

7. Schade R, Zhang X, Horacio Raúl T. Use of IgY Antibodies in Human and Veterinary Medicine. 252007. p. 213-22.

8. Han S, Zhang X, Zhao J. Production of Egg Yolk Antibody (IgY) against Recombinant Canine Parvovirus VP2 Protein. Acta Scientiae Veterinariae. $2012 ; 40$.

9. Zhang X, Chen H, Tian Z, Chen S, Schade R. Chicken monoclonal IgY antibody: a novel antibody development strategy. Avian Biol Res. 2010;3(3):97-106.

10. Lee W, Syed Atif A, Tan SC, Leow CH. Insights into the chicken IgY with emphasis on the generation and applications of chicken recombinant monoclonal antibodies. J Immunol Methods. 2017;447:71-85.

11. Indran IR, Liang RLZ, Min TE, Yong E-L. Preclinical studies and clinical evaluation of compounds from the genus Epimedium for osteoporosis and bone health. Pharmacology and Therapeutics. 2016;162.

12. Chen HX, He F, Sun Y, Luo Y, Qiu HJ, Zhang XY, et al. Generation and characterization of chicken-sourced single-chain variable fragments (scFvs) against porcine interferon-gamma (pIFN-gamma). J Immunoass Immunochem. 2015;36(1):27-44.

13. Xu J, Guo HC, Wei YQ, Dong H, Han SC, Ao D, et al. Self-assembly of virus-like particles of canine parvovirus capsid protein expressed from Escherichia coli and application as virus-like particle vaccine. Appl Microbiol Biotechnol. 2014;98(8):3529-38.

14. Hansel T, Kropshofer H, Singer T, Mitchell J, George A. The safety and side effects of monoclonal antibodies. Nature reviews Drug discovery. 2010;9:32538.

15. Dimitrov DS. Therapeutic antibodies, vaccines and antibodyomes. mAbs. 2010;2(3):347-56.

16. Dal Ferro M, Rizzo S, Rizzo E, Marano F, Luisi I, Tarasiuk O, et al. Phage Display Technology for Human Monoclonal Antibodies. Methods in molecular biology (Clifton, NJ). 2019;1904:319 - 38.

17. Supuran CT. Agents for the prevention and treatment of age-related macular degeneration and macular edema: a literature and patent review. Expert Opin Ther Pat. 2019;29(10):761-7.

18. Lee CH, Lee YC, Lee YL, Leu SJ, Lin LT, Chen CC, et al. Single Chain Antibody Fragment against Venom from the Snake Daboia russelii formosensis. Toxins. 2017;9(11). 
19. Schade R, Calzado EG, Sarmiento R, Chacana PA, Porankiewicz-Asplund J, Terzolo HR. Chicken egg yolk antibodies (IgY-technology): a review of progress in production and use in research and human and veterinary medicine. Alternatives to laboratory animals: ATLA. 2005;33(2):129-54.

20. Pereira EPV, van Tilburg MF, Florean E, Guedes MIF. Egg yolk antibodies (IgY) and their applications in human and veterinary health: A review. Int Immunopharmacol. 2019;73:293-303.

21. Sheng L, He Z, Chen J, Liu Y, Ma M, Cai Z. The impact of N-glycosylation on conformation and stability of immunoglobulin Y from egg yolk. Int J Biol Macromol. 2017;96:129-36.

22. Gilgunn S, Millan S, Wormald M, Zapatero Rodríguez J, Conroy P, O’Kennedy R, et al. Comprehensive N-Glycan Profiling of Avian Immunoglobulin Y. PLOS ONE. 2016;11:e0159859.

23. Waldmann H. Human Monoclonal Antibodies: The Benefits of Humanization. Methods in molecular biology. (Clifton NJ). 2019;1904:1-10.

24. Choi J, Kim M, Lee J, Seo Y, Ham Y, Lee J, et al. Antigen-binding affinity and thermostability of chimeric mouse-chicken IgY and mouse-human IgG antibodies with identical variable domains. Sci Rep. 2019;9(1):19242.

25. Jain NK, Sahni N, Kumru OS, Joshi SB, Volkin DB, Russell Middaugh C. Formulation and stabilization of recombinant protein based virus-like particle vaccines. Adv Drug Deliv Rev. 2015;93:42-55. 